\title{
Barriers to timely diagnosis of interstitial lung disease in the real world: the INTENSITY survey
}

\author{
Gregory P. Cosgrove ${ }^{1,2^{*}}$, Pauline Bianchi ${ }^{3}$, Sherry Danese ${ }^{4}$ and David J. Lederer ${ }^{2,5}$
}

\begin{abstract}
Background: The diagnosis of idiopathic pulmonary fibrosis (IPF) and other interstitial lung diseases (ILD) presents significant clinical challenges. To gain insights regarding the diagnostic experience of patients with ILD and to identify potential barriers to a timely and accurate diagnosis, we developed an online questionnaire and conducted a national survey of adults with a self-reported diagnosis of ILD.

Methods: A pre-specified total of 600 subjects were recruited to participate in a 40-question online survey. E-mail invitations containing a link to the survey were sent to 16427 registered members of the Pulmonary Fibrosis Foundation. Additionally, an open invitation was posted on an online forum for patients and caregivers (www.inspire.com). The recruitment and screening period was closed once the pre-defined target number of respondents was reached. Eligible participants were adult U.S. residents with a diagnosis of IPF or a non-IPF ILD.

Results: A total of 600 eligible respondents met the eligibility criteria and completed the survey. Of these, 55\% reported $\geq 1$ misdiagnosis and 38\% reported $\geq 2$ misdiagnoses prior to the current diagnosis. The most common misdiagnoses were asthma (13.5\%), pneumonia (13.0\%), and bronchitis (12.3\%). The median time from symptom onset to current diagnosis was 7 months (range, $0-252$ months), with $43 \%$ of respondents reporting a delay of $\geq 1$ year and 19\% reporting a delay of $\geq 3$ years. Sixty-one percent of respondents underwent at least one invasive diagnostic procedure.

Conclusions: While a minority of patients with ILD will experience an appropriate and expedient diagnosis, the more typical diagnostic experience for individuals with ILD is characterized by considerable delays, frequent misdiagnosis, exposure to costly and invasive diagnostic procedures, and substantial use of healthcare resources. These findings suggest a need for physician education, development of clinical practice recommendations, and improved diagnostic tools aimed at improving diagnostic accuracy in patients with ILD.
\end{abstract}

Keywords: Interstitial lung disease, Idiopathic pulmonary fibrosis, Diagnosis

\section{Background}

Interstitial lung disease (ILD) encompasses a broad and diverse group of diffuse parenchymal lung disorders that are characterized by alveolar inflammation, fibrosis, and other cellular changes. Of the more than 150 recognized ILDs, idiopathic pulmonary fibrosis (IPF) is among the most common and the most lethal $[1,2]$. The clinical course is characterized by progressive exertional dyspnea and a decline in pulmonary function that severely limits

\footnotetext{
* Correspondence: cosgroveg@njhealth.org

${ }^{1}$ National Jewish Health, 1400 Jackson Street, Denver, CO 80206, USA

${ }^{2}$ Pulmonary Fibrosis Foundation, 230 E. Ohio Street, Suite 500, Chicago, IL 60611, USA

Full list of author information is available at the end of the article
}

routine physical activity [3]. Two recently approved therapies have been shown to attenuate the decline in pulmonary function $[4,5]$; however, neither agent is a cure and neither agent improves lung function, symptoms, or quality of life. Timely and accurate diagnosis could potentially improve outcomes by permitting earlier initiation of antifibrotic therapy, avoiding exposure to harmful or ineffective treatments, and improving access to important treatments that improve quality of life, such as supplemental oxygen and pulmonary rehabilitation [6].

The diagnosis of IPF is challenging for even the most experienced clinicians. Accurate diagnosis requires careful exclusion of alternative etiologies and skillful 
integration of findings from clinical, radiologic, and pathologic exams [3, 7]. Current diagnostic guidelines define characteristic radiologic and histopathologic features that suggest a diagnosis of IPF [3]; however, high resolution computed tomography (HRCT) scans and lung biopsies frequently exhibit mixed or discordant patterns, and findings in patients with IPF and other ILDs are often marked by subtle differences [7-9]. Studies evaluating diagnostic agreement among pulmonologists, radiologists, and pathologists have reported only modest inter-observer agreement, even among expert observers [7, 10, 11]. Additionally, early diagnosis is further complicated by the insidious onset and nonspecific nature of the initial symptoms, which patients often initially attribute to age or deconditioning, leading to a delay in seeking medical attention [12].

To gain insights regarding the diagnostic experience of patients with ILD and identify potential barriers to a timely and accurate diagnosis, we developed a structured online questionnaire and conducted a national survey of adults with a self-reported diagnosis of ILD.

\section{Methods}

A structured online survey (The INTENSITY Survey) was developed to collect data on the diagnostic experiences of adults with ILD. The quantitative survey comprised 40 defined-choice and open-ended questions related to the following topics: demographics, current and prior diagnoses, initial onset and duration of symptoms, the number and type of physicians consulted, diagnostic tests and procedures, medication history, comorbidities, and quality of life. For the latter, respondents were asked to indicate the degree to which they agreed or disagreed with a series of statements designed to elicit information on the following domains: emotional, physical, financial, personal, and professional (the full survey is available in Additional file 1).

E-mail invitations containing a link to the online survey were sent to 16427 registered members of the Pulmonary Fibrosis Foundation, a nonprofit advocacy, education, and research organization dedicated to serving people living with pulmonary fibrosis, their caregivers, and healthcare professionals. Additionally, an open invitation was posted on the website of Inspire ${ }^{\mathrm{Tw}}$ (www.inspire.com), an online health community and support network designed to connect patients and caregivers with common interests. Survey participants were offered the choice of a nominal honorarium in the form of a $\$ 20$ gift card or a donation in the equivalent amount to the Pulmonary Fibrosis Foundation. The recruitment and screening period closed once the pre-defined target of 600 eligible respondents completed the survey.

Eligible participants were U.S. residents with a diagnosis of IPF or a non-IPF ILD (a full list of ILD diagnoses is available in Additional file 1). At the start of their online participation, prospective participants were informed about the content and purpose of the survey and advised that their responses would be anonymous. Screening for eligibility was accomplished by means of three online screening questions which prospective participants were required to complete prior to gaining access to the research questions.

The survey was conducted in full compliance with the American Association for Public Opinion Research best practices for research, including the reporting of outcome measures [13]. Completed survey results were tabulated by an independent research organization (Outcomes Insights Inc., Thousand Oaks, CA). Data are summarized descriptively and presented as the median (range) for continuous variables and number and percentage of respondents for categorical variables.

\section{Results}

\section{Survey response}

The survey response metrics are summarized in Fig. 1. Between August 14 and August 26, 2015, a total of 1152 individuals entered the online survey and initiated screening. Of these, 414 did not meet eligibility criteria, 69 did not complete screening, and 69 chose not to participate, resulting in a total of 600 respondents from 47 states who completed the survey. Among those who completed the survey, 503 (83.8\%) were registered members of the Pulmonary Fibrosis Foundation and 97 (16.2\%) were recruited through the open invitation on the Inspire ${ }^{\mathrm{mt}}$ online patient forum.

\section{Demographics and respondent characteristics}

Demographics and respondent characteristics are summarized in Table 1. There was an equal distribution of male and female respondents; the median age was 69 years (range, 16-91) in males and 62.5 years (21-89) in females. IPF was the most common diagnosis (46.5\%), followed by ILD (32.5\%), non-specific interstitial pneumonia (15.5\%), hypersensitivity pneumonitis (4.5\%), and sarcoidosis (1.0\%). More than two-thirds (70.1\%) of respondents were diagnosed within the last 5 years. The most commonly reported comorbid conditions were gastroesophageal reflux disease (43.3\%), sleep apnea (22.3\%), allergy (20.7\%), and cardiovascular disease (20.3\%).

\section{Diagnostic experience}

Survey results indicated that most respondents experienced a gradual onset of symptoms, which typically included shortness of breath (77.2\%), cough (53.0\%), and fatigue $(38.0 \%)$ (Table 2 ). The median reported time from initial onset of symptoms to the first doctor visit was 3 months, with $72 \%$ of respondents indicating that they initially attributed some or all of their respiratory 


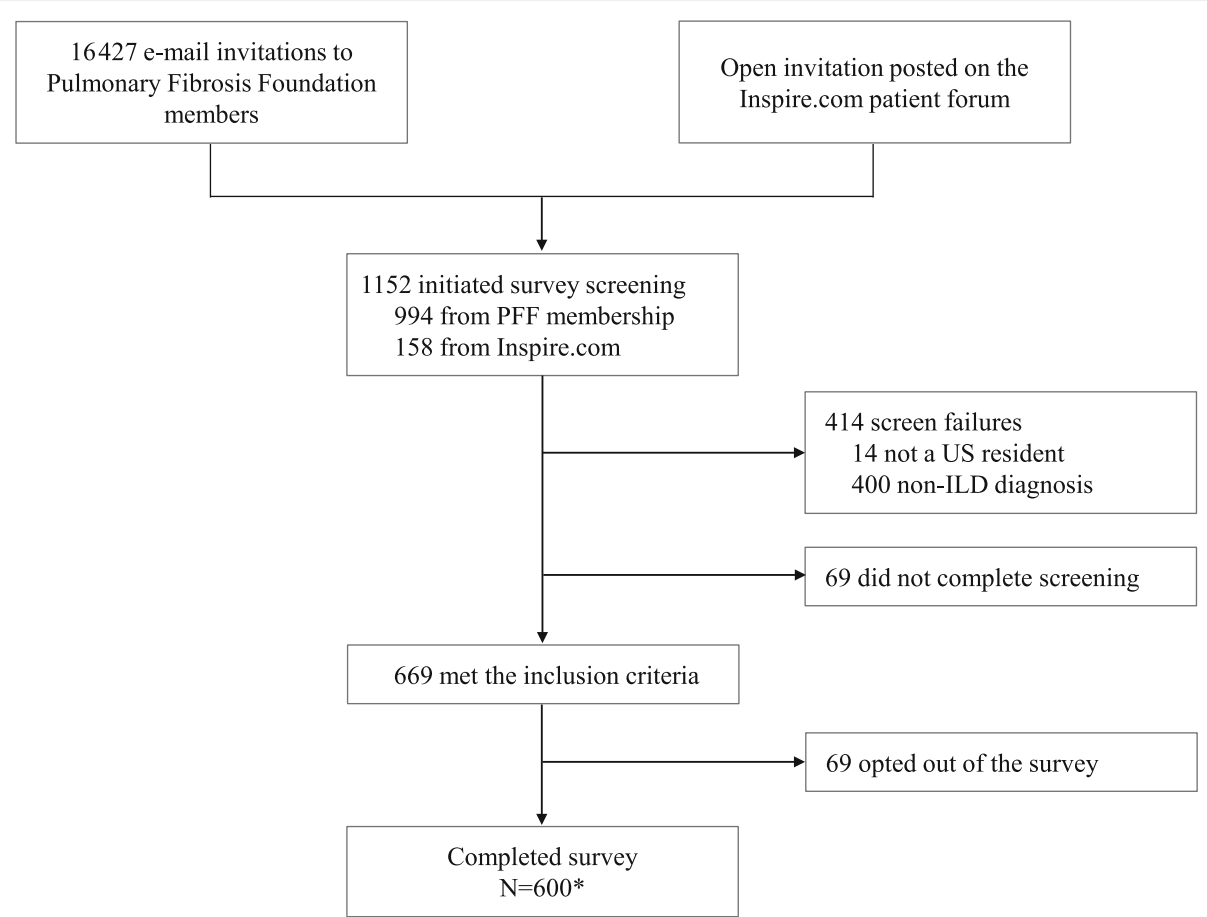

Fig. 1 Survey response summary. Includes 503 respondents from the Pulmonary Fibrosis Foundation membership and 97 respondents from the open invitation posted on the Inspire.com patient forum

symptoms to advancing age. Nearly all survey participants initially consulted a primary care physician; of these, $27.8 \%$ were referred to a specialist after the first visit, but $30.4 \%$ reported $\geq 4$ visits to a primary care physician prior to being referred to a specialist (Table 2).

More than half of all respondents (55\%) reported at least one misdiagnosis and more than one-third (38\%) reported $\geq 2$ misdiagnoses prior to the current diagnosis (Fig. 2a). Among those who were initially misdiagnosed, the median time between the initial misdiagnosis and the final diagnosis was 11 months, with 34\% reporting a delay of $\geq 2$ years (Fig. 2b). The most common misdiagnoses were asthma (13.5\%), pneumonia (13.0\%), and bronchitis (12.3\%) (Fig. 3).

The median time from initial onset of symptoms to final diagnosis was 7 months (range, 0-252). Forty-three percent of respondents reported a delay of $\geq 1$ year between the initial onset of symptoms and the final diagnosis, and nearly one in five reported experiencing a delay of $\geq 3$ years (Fig. 4). Among those who underwent an invasive diagnostic procedure, the median time from symptom onset to final diagnosis was 9 months (range, 0-252).

The majority of respondents (75\%) consulted at least three physicians prior to receiving their current diagnosis. Commonly reported diagnostic tests and procedures included spirometry, chest x-ray, oximetry, computed tomography (CT)/HRCT, and the six-minute walk test (Fig. 5). Imaging studies, exercise tests, and spirometry were commonly repeated multiple times prior to the final diagnosis. Additionally, $61 \%$ reported undergoing an invasive diagnostic procedure, including $43.3 \%$ who had a surgical lung biopsy and $35.7 \%$ who had at least one bronchoscopy.

A substantial majority of respondents $(88 \%)$ indicated that their final diagnosis was made by a pulmonologist, with $34.7 \%$ reporting that the diagnosis was made by a physician at a nationally recognized ILD center. Consulting a specialist with expertise in interstitial lung disease was cited by $68 \%$ of respondents as the most important contributing factor in obtaining a clear diagnosis.

Systemic corticosteroids were prescribed to $21.8 \%$ of survey participants during the diagnostic evaluation. Among those with a self-reported diagnosis of IPF, $21.1 \%$ reported prior treatment with systemic corticosteroids. Other commonly prescribed medications during the interval between initial medical consultation and the current diagnosis were antibiotics (32.0\%), salmeterol/ fluticasone (17.8\%), N-acetylcysteine (13.5\%), omeprazole (11.8\%), and albuterol (11.6\%).

\section{Quality of life}

Most respondents indicated that the diagnostic process had an adverse impact on their quality of life (Fig. 6). More than 90\% reported that the time required to obtain an accurate diagnosis had a meaningful effect on the amount of time spent with friends and family. Nearly half of all respondents reported difficulty finding time to 
Table 1 Respondent characteristics

\begin{tabular}{|c|c|c|c|}
\hline Characteristic & $\begin{array}{l}\text { Total } \\
(N=600)\end{array}$ & $\begin{array}{l}\text { IPF } \\
(N=279)\end{array}$ & $\begin{array}{l}\text { Non-IPF } \\
(N=321)\end{array}$ \\
\hline \multicolumn{4}{|l|}{ Median age (range), years } \\
\hline Male & $69(16-92)$ & $70(29-92)$ & $66(16-83$ \\
\hline Female & $62(21-94)$ & $64(21-84)$ & $61(30-94)$ \\
\hline \multicolumn{4}{|l|}{ Gender, n (\%) } \\
\hline Male & $300(50.0)$ & $164(58.8)$ & $136(42.4)$ \\
\hline Female & $300(50.0)$ & $115(41.2)$ & $185(57.6)$ \\
\hline \multicolumn{4}{|l|}{ Diagnosis, n (\%) } \\
\hline Idiopathic pulmonary fibrosis & $279(46.5)$ & $279(100.0)$ & $0(0.0)$ \\
\hline Interstitial lung disease ${ }^{*}$ & $195(32.5)$ & $0(0.0)$ & $195(60.7)$ \\
\hline Non-specific interstitial pneumonia & $93(15.5)$ & $0(0.0)$ & $93(29.0)$ \\
\hline Hypersensitivity pneumonitis & $27(4.5)$ & $0(0.0)$ & $27(8.4)$ \\
\hline Sarcoidosis & $6(1.0)$ & $0(0.0)$ & $6(1.9)$ \\
\hline \multicolumn{4}{|l|}{ Time since diagnosis, n (\%) } \\
\hline$\leq 6$ months & $32(5.3)$ & $18(6.5)$ & $14(4.4)$ \\
\hline$>6$ months to 1 year & $59(9.8)$ & $33(11.8)$ & $26(8.1)$ \\
\hline$>1$ to 2 years & $126(21.0)$ & $61(21.9)$ & $65(20.2)$ \\
\hline$>2$ to 5 years & $204(34.0)$ & $93(33.3)$ & $111(34.6)$ \\
\hline$>5$ to 10 years & $127(21.2)$ & $57(20.4)$ & $70(21.8)$ \\
\hline$>10$ years & $52(8.6)$ & $17(6.1)$ & $35(10.9)$ \\
\hline \multicolumn{4}{|l|}{ Comorbid conditions, n (\%) } \\
\hline Gastroesophageal reflux disease & $260(43.3)$ & $115(41.2)$ & $145(45.2)$ \\
\hline Sleep apnea & $134(22.3)$ & $68(24.4)$ & $66(20.6)$ \\
\hline Allergy & $124(20.7)$ & $52(18.6)$ & $72(22.4)$ \\
\hline Cardiovascular disease & $122(20.3)$ & $59(21.1)$ & $63(19.6)$ \\
\hline Pulmonary hypertension & $103(17.2)$ & $37(13.3)$ & $66(20.6)$ \\
\hline Other pulmonary conditions ${ }^{\dagger}$ & $356(59.3)$ & $156(55.9)$ & $200(62.3)$ \\
\hline \multicolumn{4}{|l|}{ Family history of ILD, n (\%) } \\
\hline Yes & $102(17.0)$ & $54(19.4)$ & $48(15.0)$ \\
\hline No & $498(83.0)$ & $225(80.6)$ & $273(85.0)$ \\
\hline
\end{tabular}

*Includes interstitial lung disease due to an autoimmune or connective tissue disease, respiratory bronchiolitis-interstitial lung disease, desquamative interstitial pneumonia, cryptogenic organizing pneumonia, lymphoid interstitial pneumonia, idiopathic pleuroparenchymal fibroelastosis, interstitial lung disease due to occupational exposure, radiation therapy-induced interstitial lung disease, and drug-related interstitial lung disease

${ }^{\dagger}$ Includes chronic obstructive pulmonary disease, asthma, chronic bronchitis/bronchiolitis, bronchiectasis, emphysema, pneumonia, cystic fibrosis, pulmonary edema, lung cancer, and tuberculosis

ILD = interstitial lung disease; IPF = idiopathic pulmonary fibrosis

attend doctor visits, with $28 \%$ reporting that time spent attending medical appointments and undergoing diagnostic procedures contributed at least in part to their decision to apply for disability benefits or retire. Diagnostic tests and procedures were commonly characterized as physically stressful, and more than $80 \%$ of respondents reported some degree of emotional stress due to the ongoing uncertainty regarding their diagnosis.

\section{Discussion}

We have shown that the diagnostic experience for a substantial percentage of U.S. adults with ILD is characterized by repeated physician visits, multiple and often repeated diagnostic tests, frequent misdiagnosis, exposure to invasive procedures, and treatment with multiple therapies. Despite considerable progress in the understanding of IPF and other ILDs, these findings suggest a dichotomy in the diagnostic experience, with some patients receiving timely and accurate diagnoses, but a much larger proportion of patients experiencing significant challenges. This latter group the focus of the remaining discussion.

We identified several potential obstacles to a timely and accurate diagnosis. First, the common and nonspecific nature of the symptoms often leads patients to 
Table 2 Initial presentation

\begin{tabular}{|c|c|}
\hline & $\begin{array}{l}\text { Respondents } \\
(N=600)\end{array}$ \\
\hline \multicolumn{2}{|l|}{ Initial symptoms, n (\%) } \\
\hline Shortness of breath & $463(77.2)$ \\
\hline Cough & $318(53.0)$ \\
\hline Fatigue/weakness & $228(38.0)$ \\
\hline Chest discomfort & $104(17.3)$ \\
\hline Unexplained weight loss & $40(6.7)$ \\
\hline Loss of appetite & $33(5.5)$ \\
\hline Pneumonia & $13(2.2)$ \\
\hline Other & $58(9.7)$ \\
\hline \multicolumn{2}{|c|}{ Time between initial symptoms and first doctor visit, $\mathrm{n}(\%)$} \\
\hline $0-5$ months & $356(59.3)$ \\
\hline 6-11 months & $101(16.8)$ \\
\hline $12-23$ months & $64(10.7)$ \\
\hline 24-35 months & $42(7.0)$ \\
\hline 36-47 months & $14(2.3)$ \\
\hline$\geq 48$ months & $23(3.8)$ \\
\hline \multicolumn{2}{|c|}{ Initial medical consultation, n (\%) } \\
\hline Primary care physician & $533(88.8)$ \\
\hline Specialist & $67(11.2)$ \\
\hline \multicolumn{2}{|c|}{ Primary care visits prior to referral, $\mathrm{n}(\%)^{*}$} \\
\hline 1 & $148(27.8)$ \\
\hline 2 & 99 (18.6) \\
\hline 3 & 99 (18.6) \\
\hline 4 & $35(6.6)$ \\
\hline$>4$ & $127(23.8)$ \\
\hline Do not recall & $23(4.3)$ \\
\hline No specialist referral & $2(0.4)$ \\
\hline
\end{tabular}

${ }^{*} N=533$ dismiss initial symptoms and forego seeking medical attention. From the physician's perspective, the typical presenting symptoms are more likely to be an indication of a common respiratory or cardiovascular condition, thus potentially leading to delays while such diagnoses are ruled out. Second, due to the diagnostic complexity of IPF and other ILDs, patients often undergo multiple diagnostic tests and procedures, including spirometry, serology, chest x-ray, HRCT, bronchoscopy, exercise tests, and surgical lung biopsy. Delays related to scheduling and availability, interpretation of test results, and the need to repeat certain tests and procedures contribute to the lengthy diagnostic process which, in turn, is reported to impose a considerable burden on patients and their caregivers. Indeed, a novel finding of the survey was the observation that $28 \%$ of respondents reported that the burden imposed by the diagnostic process was a factor in their decision to apply for disability benefits or retire. While further research is necessary to quantify the economic impact in terms of lost wages and reduced retirement benefits for both the patient and the primary caregiver, the observation that more than one-quarter of respondents reported that the diagnostic process was a contributing factor in the decision to retire or file for disability benefits suggests a need for meaningful improvements in diagnostic methods, timeliness, and accuracy. Finally, limited access or proximity to tertiary care-level expertise was commonly identified as an obstacle to obtaining a timely and accurate diagnosis. Notably, $68 \%$ of respondents cited referral to a subspecialist with expertise in ILD as the most important contributing factor to obtaining a clear diagnosis.

For patients with IPF who experience continued progression of disease during the diagnostic process, the consequences of a delay or misdiagnosis are particularly grave. Several studies have shown that reductions in forced vital capacity as small as 5-10\% over 6 months are associated with a significant increase in the risk of
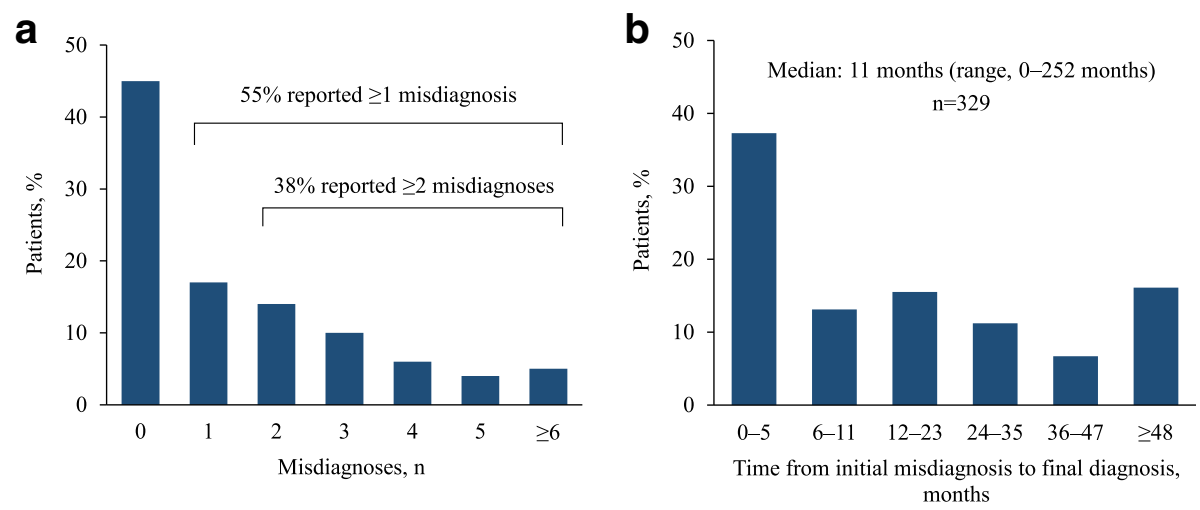

Fig. 2 Reported frequency of misdiagnosis (a) and time from initial misdiagnosis to final diagnosis (b) 


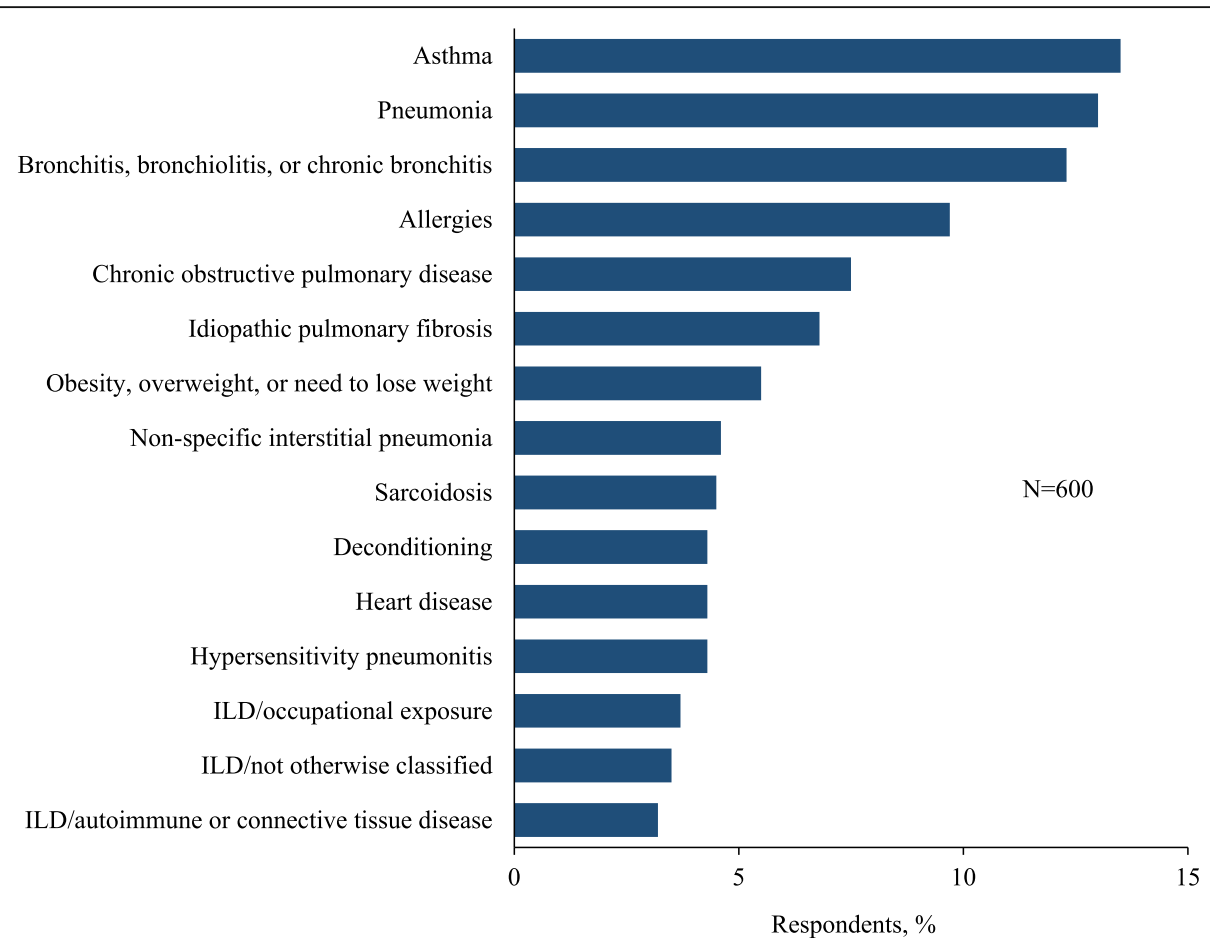

Fig. 3 Most commonly reported misdiagnoses (reported by $>3 \%$ of respondents). ILD = interstitial lung disease

death, [14-19] and delayed referral to subspecialty care has been shown to confer an increased risk of death in patients with IPF [20]. Additionally, a delay in obtaining an accurate diagnosis can delay evaluation for lung transplantation, potentially resulting in the loss of eligibility due to advanced age or frailty. Lastly, while the typical presenting symptoms of IPF mimic those of many other common pulmonary and cardiovascular disorders, they are uniquely unresponsive to agents frequently used in the treatment of other ILDs. Misdiagnosis therefore carries the added risk of exposure to

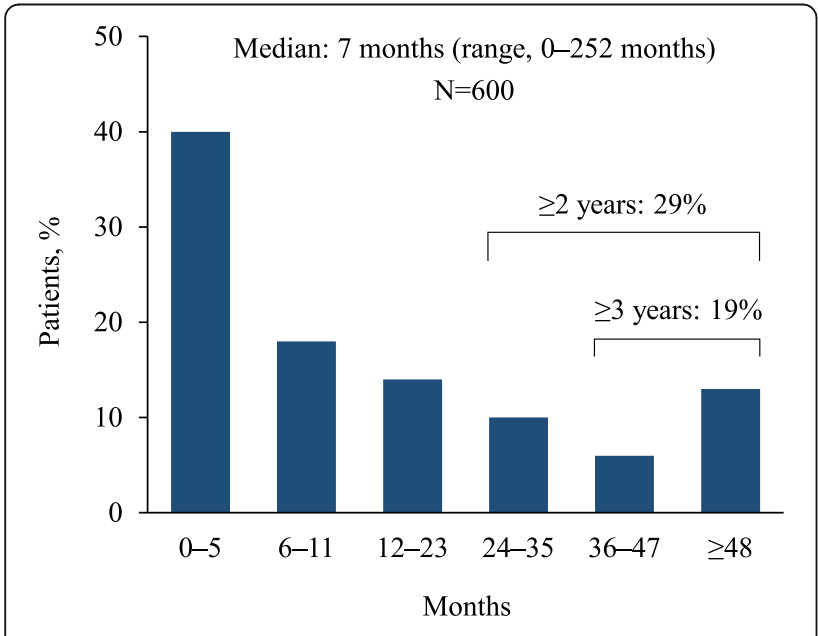

Fig. 4 Time from initial onset of symptoms to final diagnosis ineffective or harmful therapies. In the present survey, one in five respondents with a current diagnosis of IPF reported prior treatment with a systemic corticosteroid-a potentially harmful therapy that carries a strong negative recommendation in the current international treatment guidelines $[3,21]$.

The findings of the survey are generally consistent with observations from two prior surveys of patients with pulmonary fibrosis [12, 22]. In a 2007 U.S. survey of patients with a physician-confirmed diagnosis of IPF, $55 \%$ of respondents reported a delay of at least 1 year between the initial onset of symptoms and a final diagnosis, and 38\% reported consulting $\geq 3$ physicians before a diagnosis of pulmonary fibrosis was established [22]. The most commonly reported misdiagnoses were bronchitis, asthma, and chronic obstructive pulmonary disease. A subsequent survey of IPF patients in five European countries reported similar findings [12]. Fifty-eight percent of patients experienced a delay of more than 1 year between initial presentation and a confirmed diagnosis of IPF, and more than half reported consulting $\geq 3$ physicians before receiving a final diagnosis. The median time from initial presentation to confirmed diagnosis was 1.5 years. Asthma, chronic obstructive pulmonary disease, and pneumonia were the most commonly reported initial misdiagnoses. In the European survey, participants identified improved diagnostic techniques and improved access to tertiary care centers with expertise in ILD as the most common unmet needs. 


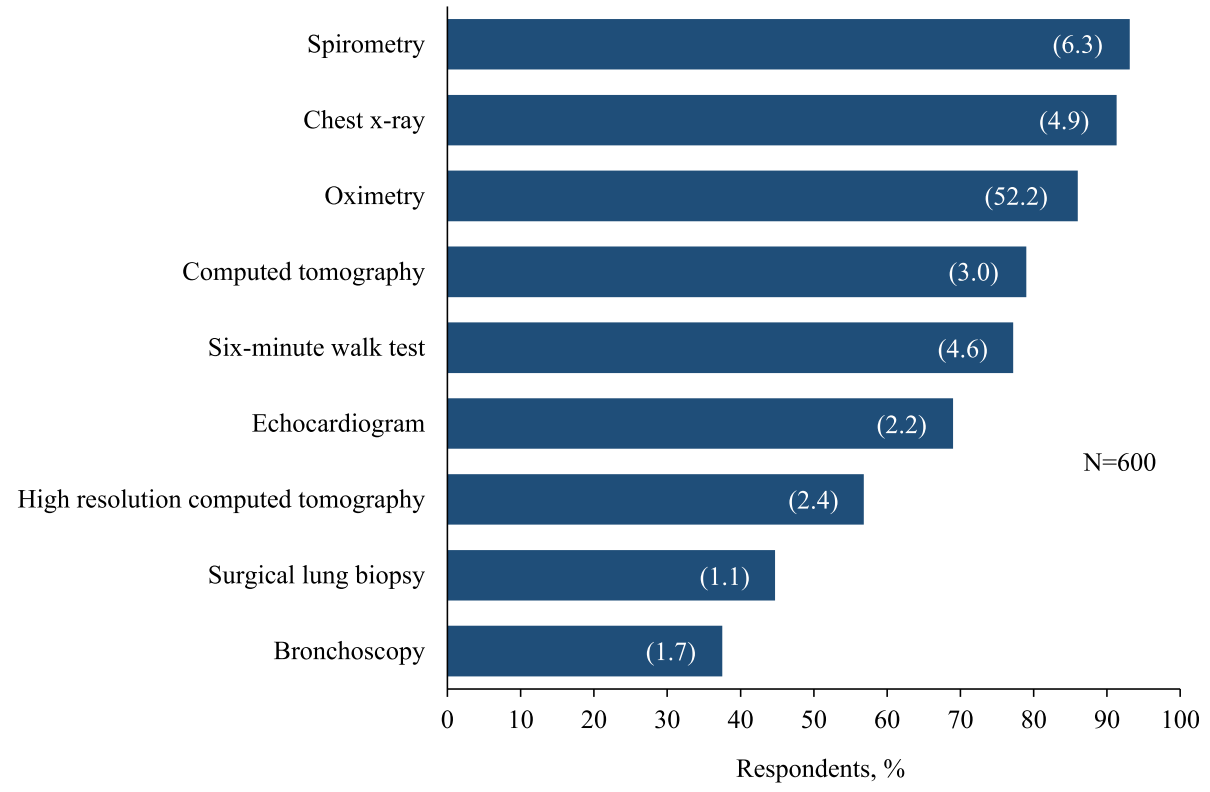

Fig. 5 Diagnostic investigations. Data are presented as percentage of respondents, with the mean frequency per respondent in parentheses. The mean frequency is based on the number of respondents reporting the corresponding diagnostic investigation

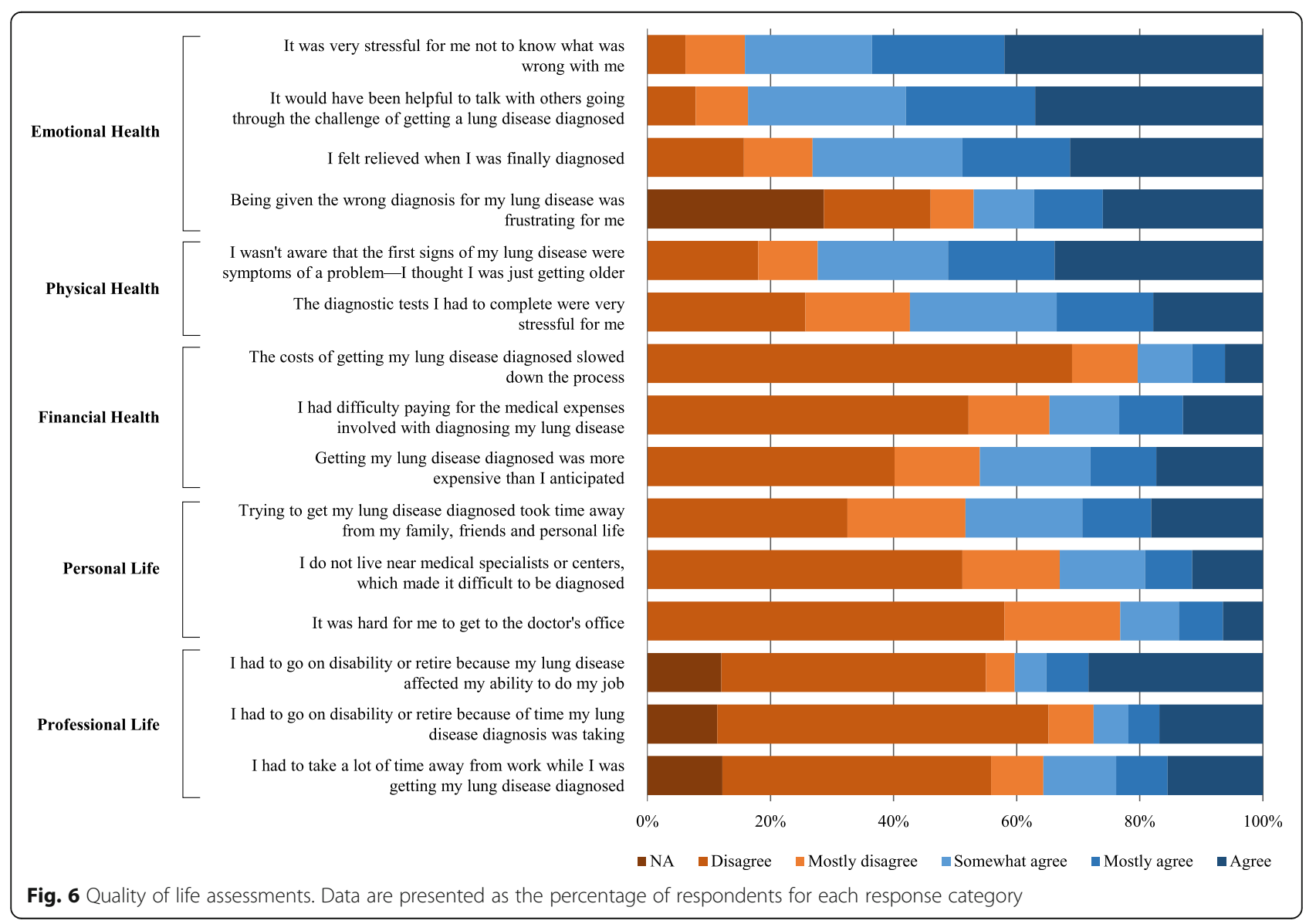


In contrast to the previous surveys, more than $70 \%$ of respondents in the present survey were diagnosed after the 2011 publication of the current IPF diagnostic guidelines [3]. Comparison of results from the present survey with those from surveys conducted prior to the 2011 guidelines suggest that the new guidelines have had a marginal impact on the self-reported diagnostic experience of individuals with IPF. A lower proportion of respondents in the present survey experienced $\mathrm{a} \geq 1$-year delay in obtaining a clear diagnosis compared with the previous U.S. and European surveys $(42 \%$ vs. $55 \%$ and $58 \%$, respectively); however, a higher proportion of respondents in the present survey consulted $\geq 3$ physicians compared with the previous surveys $(75 \%$ vs. $38 \%$ and $55 \%$, respectively). More than half of all respondents in both the current survey and the prior U.S. survey reported at least one misdiagnosis, with no evidence to suggest a change in the reported frequency of misdiagnosis following the revision of the guidelines. Additionally, while the current guidelines indicate that a pattern of usual interstitial pneumonia on HRCT is sufficient for a diagnosis of IPF under appropriate clinical circumstances, a surgical lung biopsy was performed in roughly half of all participants in both U.S. surveys, suggesting that clinical and radiological findings were inconclusive in as many as $50 \%$ of respondents. For those with severe disease or comorbidities, the increased risk might preclude surgery, resulting in an uncertain diagnosis and an inability to determine the optimal therapeutic intervention.

The results of the survey should be interpreted in the context of certain limitations. The data were collected from a non-random sample of adults living with ILD, the majority of whom were registered members of a national patient advocacy and support organization. The degree to which the findings may be generalized to a broader population is therefore unknown. Furthermore, prevalent rather than incident cases were sampled, which typically captures subjects with lesser disease severity and slower progression. Therefore, the survey might not have captured the most extreme cases of late diagnosis, as those diagnosed close to death were less likely to engage the Pulmonary Fibrosis Foundation or participate in the study. Additionally, subjects who had an unremarkable diagnostic experience or those in certain demographic groups might have also been less likely to participate in an online diagnostic survey, thereby introducing the potential for error due to nonresponse bias. Diagnoses and diagnostic procedures were self-reported by respondents and were not confirmed by medical records; accordingly, responses to these and other questions related to medical terms and procedures are subject to error due to misinformation bias. Finally, responses to questions requiring an accurate memory of historical details are subject to error due to recall bias. Despite these limitations, we believe the results of the survey provide important insights that will inform efforts to improve the diagnostic experience of patients with IPF and other ILDs.

\section{Conclusions}

A nationwide survey of adults with ILD found that the diagnostic process is characterized by considerable delays, frequent misdiagnosis, exposure to costly and invasive diagnostic procedures, and substantial use of healthcare resources. Moreover, the experience is associated with meaningful adverse emotional, physical, social, and professional consequences for patients and their caregivers. These findings underscore the need for physician education, practical clinical guidelines, and improved diagnostic tools that increase the speed and accuracy of diagnosis and facilitate early therapeutic intervention.

\section{Additional files}

\section{Additional file 1: INTENSITY Survey Questions and Response Data.} (DOCX $605 \mathrm{~kb}$ )

\section{Abbreviations}

CT: Computed tomography; CTD: Connective tissue disease; FVC: Forced vital capacity; HRCT: High resolution computed tomography; ILD: Interstitial lung disease; IPF: Idiopathic pulmonary fibrosis

\section{Acknowledgments}

We would like to acknowledge the efforts of the study participants and their care givers. It is through their continued participation that we will better understand the impact of pulmonary fibrosis on the lives of those with the disease and improve the care of all those affected. Additionally, we thank the Pulmonary Fibrosis Foundation for collaboration and recruitment assistance, the American Lung Association for recruitment assistance, Jeanne Loboda for project management support, and Kenneth Glasscock for medical writing and editorial assistance.

\section{Funding}

The survey was sponsored by Veracyte Inc. and conducted in collaboration with the Pulmonary Fibrosis Foundation.

\section{Availability of data and materials}

The full INTENSITY Survey and response data are available in Additional file 1.

\section{Authors' contributions}

GPC, PB, SD, and DJL contributed to the conception and design of the survey, the analysis and interpretation of original data, and the preparation of the manuscript. All authors vouch for the integrity of the data and the accuracy of the report. Each author had full access to data, and no limits were placed on the content of the report. All authors critically reviewed the manuscript and approved the final draft.

\section{Ethics approval and consent to participate}

The research would be considered exempt under applicable federal regulations (45 CFR 46.101b) based on the American Association for Public Opinion Research policy participant category. Our respondents were active participants in that they opted-in to participate in the research based on their choice to continue from the screener to the survey. Moreover, our respondents were informed that their participation "gives you an opportunity to share your experiences when being diagnosed with PF". 


\section{Consent for publication}

Not applicable.

\section{Competing interests}

GPC has served as an investigator or collaborator in industry-sponsored clinical trials (Boehringer Ingelheim, Bristol Myers Squibb, Fibrogen, Genentech, Gilead Sciences, Global Blood Therapeutics, and Intermune) and as an advisor to Boehringer Ingelheim, InterMune, Genentech, and Global Blood Therapeutics; he currently serves as the Chief Medical Officer of the Pulmonary Fibrosis Foundation. PB is an employee of Veracyte. SD reports no competing interests. DJL served as a consultant for Boehringer Ingelheim, Genentech, Gilead Sciences, Galapagos, Patara, Phillips Respironics, the Pulmonary Fibrosis Foundation, and Veracyte, and has received institutional research grants from Bayer, Boehringer Ingelheim, Fibrogen, Gilead Sciences, and Global Blood Therapeutics.

\section{Publisher's Note}

Springer Nature remains neutral with regard to jurisdictional claims in published maps and institutional affiliations.

\section{Author details}

${ }^{1}$ National Jewish Health, 1400 Jackson Street, Denver, CO 80206, USA. ${ }^{2}$ Pulmonary Fibrosis Foundation, 230 E. Ohio Street, Suite 500, Chicago, IL 60611 , USA. ${ }^{3}$ Veracyte, Inc., 6000 Shoreline Court, Suite 300, South San Francisco, CA 94080, USA. ${ }^{4}$ Outcomes Insights, Inc., 2801 Townsgate Road, Suite 30, Westlake Village, CA 91331, USA. ${ }^{5}$ Departments of Medicine and Epidemiology, Columbia University Medical Center, 622 W 168th S, PH14-101, New York, NY 10032, USA.

Received: 10 May 2017 Accepted: 8 December 2017

\section{Published online: 17 January 2018}

\section{References}

1. Flaherty KR, Toews GB, Travis WD, et al. Clinical significance of histological classification of idiopathic interstitial pneumonia. Eur Respir J. 2002;19:275-83

2. Bjoraker JA, Ryu JH, Edwin MK, et al. Prognostic significance of histopathologic subsets in idiopathic pulmonary fibrosis. Am J Respir Crit Care Med. 1998;157:199-203.

3. Raghu G, Collard HR, Egan JJ, et al. An official ATS/ERS/JRS/ALAT statement: idiopathic pulmonary fibrosis: evidence-based guidelines for diagnosis and management. Am J Respir Crit Care Med. 2011;183:788-824.

4. King TE Jr, Bradford WZ, Castro-Bernardini S, et al. A phase 3 trial of pirfenidone in patients with idiopathic pulmonary fibrosis. N Engl J Med. 2014;370:2083-92.

5. Richeldi L, du Bois RM, Raghu G, et al. Efficacy and safety of nintedanib in idiopathic pulmonary fibrosis. N Engl J Med. 2014;370:2071-82.

6. Oldham JM, Noth I. Idiopathic pulmonary fibrosis: early detection and referral. Respir Med. 2014;108:819-29.

7. Flaherty KR, King TE Jr, Raghu G, et al. Idiopathic interstitial pneumonia: what is the effect of a multidisciplinary approach to diagnosis? Am J Respir Crit Care Med. 2004;170:904-10.

8. Flaherty KR, Travis WD, Colby TV, et al. Histopathologic variability in usual and nonspecific interstitial pneumonias. Am J Respir Crit Care Med. 2001:164:1722-7.

9. Yagihashi K, Huckleberry J, Colby TV, et al. Radiologic-pathologic discordance in biopsy-proven usual interstitial pneumonia. Eur Respir J. 2016:47:1189-97.

10. Flaherty KR, Andrei AC, King TE Jr, et al. Idiopathic interstitial pneumonia: do community and academic physicians agree on diagnosis? Am J Respir Crit Care Med. 2007:175:1054-60.

11. Lynch DA, Godwin JD, Safrin S, et al. High-resolution computed tomography in idiopathic pulmonary fibrosis: diagnosis and prognosis. Am J Respir Crit Care Med. 2005;172:488-93.

12. Schoenheit G, Becattelli I, Cohen AH. Living with idiopathic pulmonary fibrosis: an in-depth qualitative survey of European patients. Chron Resp Dis. 2011:8:225-31.

13. AAPOR (1991). Code of Professional Ethics and Practices. Available at: http:// www.aapor.org/Standards-Ethics/AAPOR-Code-of-Ethics.aspx.

14. Richeldi L, Ryerson CJ, Lee JS, et al. Relative versus absolute change in forced vital capacity in idiopathic pulmonary fibrosis. Thorax. 2012;67:407-11.
15. du Bois RM, Weycker D, Albera C, et al. Ascertainment of individual risk of mortality for patients with idiopathic pulmonary fibrosis. Am J Respir Crit Care Med. 2011;184:459-66.

16. Zappala CJ, Latsi PI, Nicholson AG, et al. Marginal decline in FVC is associated with a poor outcome in idiopathic pulmonary fibrosis. Eur Respir J. 2009:35:830-6.

17. Jegal Y, Kim DS, Shim TS, et al. Physiology is a stronger predictor of survival than pathology in fibrotic interstitial pneumonia. Am J Respir Crit Care Med. 2005:171:639-44.

18. Collard HR, King TE, Bartelson BB, Vourlekis JS, Schwarz MI, Brown KK. Changes in clinical and physiologic variables predict survival in idiopathic pulmonary fibrosis. Am J Respir Crit Care Med. 2003;168:538-42.

19. Flaherty KR, Mumford JA, Murray S, et al. Prognostic implications of physiologic and radiographic changes in idiopathic interstitial pneumonia. Am J Respir Crit Care Med. 2003;168:543-8.

20. Lamas DJ, Kawut SM, Bagiella E, Philip N, Arcasoy SM, Lederer DJ. Delayed access and survival in idiopathic pulmonary fibrosis: a cohort study. Am J Respir Crit Care Med. 2011;184:842-7.

21. Idiopathic Pulmonary Fibrosis Clinical Research Network, Raghu G, Anstrom KJ, King TE Jr, Lasky JA, Martinez FJ. Prednisone, azathioprine, and Nacetylcysteine for pulmonary fibrosis. N Engl J Med. 2012;366:1968-77. Epub 2012 May 20

22. Collard HR, Tino G, Noble PW, et al. Patient experiences with pulmonary fibrosis. Respir Med. 2007:101:1350-4.

\section{Submit your next manuscript to BioMed Central and we will help you at every step:}

- We accept pre-submission inquiries

- Our selector tool helps you to find the most relevant journal

- We provide round the clock customer support

- Convenient online submission

- Thorough peer review

- Inclusion in PubMed and all major indexing services

- Maximum visibility for your research

Submit your manuscript at www.biomedcentral.com/submit
Biomed Central 